\section{Fifth International Conference Focuses on Microscopy of Semiconducting Materials}

This international conference, the fifth in a biennial series, brought a prominent area of semiconductor research sharply into focus. Based in the University of Oxford, UK, the event took place on April 6-8, 1987 and attracted about 280 delegates from 21 countries. The conference chairmen were Tony Cullis (RSRE, Malvern) and Peter Augustus (Plessey Research, Caswell). Scientific sponsorship was provided by the Institute of Physics (UK), the Royal Microscopical Society, the Materials Research Society, and the European Materials Research Society.

The scientific schedule concentrated on the important applications of microscopy and related analytical techniques to the study of semiconductors. The meeting sessions comprised 138 papers, and state-ofthe-art advances were described in areas ranging from fundamental materials investigations to electronic device work. Approximately half of the papers were presented in oral sessions; the other half were displayed as posters available for viewing throughout most of the conference period and concurrently with oral sessions. Invited papers by keynote speakers introduced important topic areas, within which contributed papers were grouped.

Following the opening address by Prof. Sir Peter Hirsch (1983 MRS Von Hippel Award winner), the first session highlighted high resolution transmission electron microscopy. A paper by J.L. Hutchison (Oxford University) demonstrated the importance of lattice and atomic imaging for the elucidation of the finest details of semiconductor interfaces. Impressive examples showing heteroepitaxial interfaces were presented. The high resolution imaging topic was developed by A. Bourret (CEN, Grenoble), who described the latest understanding of defects induced in bulk silicon by both oxygen precipitation and intrinsic processes. Other papers continued the high resolution theme with both theoretical and experimental investigations of a range of interfaces and bulk materials, forcefully demonstrating the great interest in this analytical approach.

A special session on crystal dislocations was introduced by $\mathrm{H}$. Gottschalk (Cologne University), who concentrated on developments in studies of the fine structure of dislocations in silicon. Subsequent papers expanded the area of consideration to compound semiconductors and included description of the materials effect of hydrostatic pressure.

The conference featured an extremely large amount of electron microscope work on epitaxial layer structures. An opening paper by W.L. Brown (AT\&.T Bell Laboratories, Murray Hill, NJ - 1984 MRS Von Hippel Award winner) graphically illustrated the interplay between interfacial crystallization and amorphization processes in silicon during ion bombardment. Contributed papers addressed the problems of epitaxy in both elemental and com-

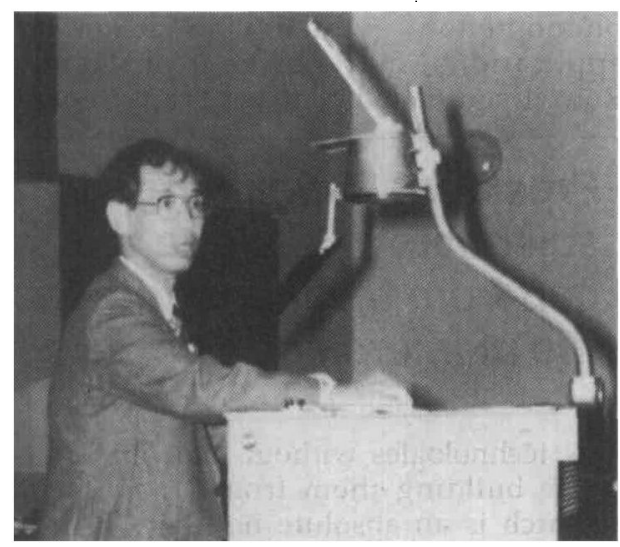

J. Matsui gives a Japanese perspective on technologically important defects in bulk gallium arsenide.

pound semiconducting materials systems. Topics receiving detailed consideration ranged from the measurement of layer strain by convergent beam electron microscopy to studies of atomic ordering in compound semiconductors. There was particular emphasis on quantum well and superlattice investigations and P.M Petroff (University of California, Santa Barbara) comprehensively demonstrated the effects of well dimensionality and described the properties of specially prepared quantum wires and quantum boxes.

The theme of superlattice studies was represented strongly in the paper by W.J. Bartels (Philips Laboratories, Eindhoven), where applications of $x$-ray diffraction methods were considered in depth. Other reported $x$-ray work ranged from specialized superlattice investigations to the characterization of bulk gallium arsenide and indium phosphide.

Energy dispersive $x$-ray microanalysis in the electron microscope had been used by many workers to determine the elemental composition of samples. This and other related analytical techniques were reviewed by M.H. Loretto (Birmingham University) with a focus on the spatial resolution and sensitivity which may be achieved. The spectacular atomic resultion provided by pulsed laser atom probe analysis was demonstrated by C.R.M. Grovenor (Oxford University), who described important advances in the application of the method.

A special session was devoted to the use of advanced scanning electron microscope techniques. L.J. Balk (Duisburg University) focused on the exploitation of acoustic wave generation by the electron beam in order to characterize materials and devices. Most other papers in this area highlighted developments in electron beam induced conductivity and cathodoluminescence studies of silicon and III-V compounds.

Due to the central importance of silicon device processing, many papers addressed studies of specific problems in this area. Following an overview by S.R. Wilson (Motorola Laboratories, Phoenix, AZ), contributed papers covered topics from developmental DRAM studies to the assessment of silicide epitaxy. There was particular interest in the characterization of buried dielectric layers formed, especially, by high-dose ion implantation. Electron beam testing of finished VLSI devices was reviewed by J.S. Wolcot (IBM Laboratories, Poughkeepsie, NY) and advances in testing techniques were described by a number of other authors. The advantages of laser beam testing of devices, a recently developed approach, were highlighted by G. Auvert (CNET, Grenoble).

The great current interest in the properties of bulk gallium arsenide ensured that many papers concentrated on this topic. J. Matsui (NEC Laboratories, Kawasaki) surveyed the characteristics of dislocations which form during the growth of gallium arsenide ingots used for device fabrication. Following papers detailed dislocationrelated effects and included studies exploiting infra-red absorption microscopy, with the determination of EL2 deep level distributions. Prof. C.B. Carter (Cornell University, Ithaca, NY) demonstrated the complexity of dislocation structures which can occur with reference to defects introduced by carefully controlled crystal deformation.

Continued

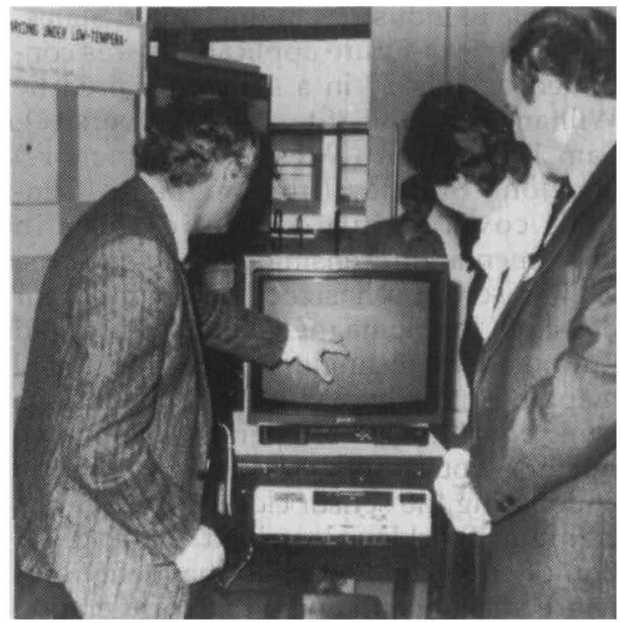

Yu. A. Ossipyan (USSR Academy of Sciences, Moscow) describes the dynamic behavior of dislocations using a videotape. 
Overall consideration of defects in bulk gallium arsenide was reserved for the last day of the conference since there was then a good interface with a special one-day workshop on this subject, which took place on April 9, 1987. The workshop was organized under the auspices of NATO and focused on the state-of-the-art in bulk gallium arsenide materials production, with particular reference to the requirements of the semiconductor industry.
A further feature of the conference was the annual RMS materials lecture, which took place on the evening of the first day. H. Rohrer (IBM Zurich Laboratories - joint winner of the 1985 Nobel Prize for physics) gave a fascinating description of the latest developments in scanning tunneling microscopy to a packed audience.

The Microscopy of Semiconducting Materials conference generated extensive interaction between semiconductor research and development workers from laboratories worldwide. The proceedings of the conference will be published later this year and will be available from: Taylor and Francis, 242 Cherry Street, Philadelphia, PA 19106-1906 USA (800-821-8312); or IOP Publishing Ltd., Techno House, Redcliffe Way, Bristol BS1 6NX, UK (0272297481).

ANTHONY G. CULLIS

\section{MATERIALS LETTERS}

\section{At Special MRS Member Rates}

As a member of the Materials Research Society, you can subscribe to Materials Letters at a special subscription rate. Only members of MRS may receive personal subscriptions to this valuable journal.*

Volume V of Materials Letters will be completed during 1987. To receive the first issue of Volume $V$ as soon as it is published this year, subscribe now by completing the form below. For your convenience, you may also subscribe to Volume VI to ensure continuation of your subscription after Volume $V$ without pause in service.

"Institutional subscriptions for libraries must be obtained through the publisher. Elsevier Science Publishers B.V...Journal Department. P.O. Box 211. 1000 AE Amsterdam. The Netherlands.

\section{MATERIALS LETTERS}

I am a member of the Materials Research Society and wish to subscribe to the following volumes of Materials Letters at the special MRS member personal subscription rate.

Check appropriate volume(s):

Volume V (current volume) @ \$20.00

Volume VI @ \$20.00

TOTAL ENCLOSED

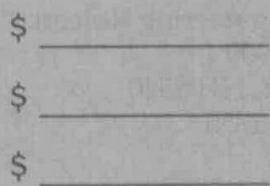

Name

Organization

Address

City State Zip/Postal Code

Country

Return subscription order with payment to: Materials Letters Subscription, Materials Research Society, 9800 McKnight Road, Suite 327, Pittsburgh, PA 15237; telephone (412) $367-3012$ 